\title{
How to include the variability of TMS responses in simulations: a speech mapping case study
}

\author{
N De Geeter ${ }^{1}$, P Lioumis ${ }^{2,3}$, A Laakso ${ }^{2,4}$, G Crevecoeur ${ }^{1}$ \\ and L Dupré ${ }^{1}$ \\ 1 Department of Electrical Energy, Systems and Automation, Ghent University, \\ Belgium \\ ${ }^{2}$ Neuroscience Center, University of Helsinki, Finland \\ 3 The BioMag Laboratory, HUS Medical Imaging Center, Helsinki University \\ Hospital, Finland \\ ${ }^{4}$ Department of Neurosurgery, Helsinki University Hospital, Finland \\ E-mail: Nele.DeGeeter@UGent.be
}

Received 26 February 2016, revised 31 August 2016

Accepted for publication 8 September 2016

Published 7 October 2016

\begin{abstract}
When delivered over a specific cortical site, TMS can temporarily disrupt the ongoing process in that area. This allows mapping of speech-related areas for preoperative evaluation purposes. We numerically explore the observed variability of TMS responses during a speech mapping experiment performed with a neuronavigation system. We selected four cases with very small perturbations in coil position and orientation. In one case (E) a naming error occurred, while in the other cases $\left(\mathrm{NE}_{\mathrm{A}, \mathrm{B}, \mathrm{C}}\right)$ the subject appointed the images as smoothly as without TMS. A realistic anisotropic head model was constructed of the subject from T1-weighted and diffusion-weighted MRI. The induced electric field distributions were computed, associated to the coil parameters retrieved from the neuronavigation system. Finally, the membrane potentials along relevant white matter fibre tracts, extracted from DTI-based tractography, were computed using a compartmental cable equation. While only minor differences could be noticed between the induced electric field distributions of the four cases, computing the corresponding membrane potentials revealed different subsets of tracts were activated. A single tract was activated for all coil positions. Another tract was only triggered for case $\mathrm{E} . \mathrm{NE}_{\mathrm{A}}$ induced action potentials in 13 tracts, while $\mathrm{NE}_{\mathrm{B}}$ stimulated 11 tracts and $\mathrm{NE}_{\mathrm{C}}$ one. The calculated results are certainly sensitive to the coil specifications, demonstrating the observed variability in this study. However, even though a tract connecting Broca's with Wernicke's area is only triggered for the error case, further research is needed on other study cases and on
\end{abstract}


refining the neural model with synapses and network connections. Caseand subject-specific modelling that includes both electromagnetic fields and neuronal activity enables demonstration of the variability in TMS experiments and can capture the interaction with complex neural networks.

Keywords: electric field, membrane potential, speech mapping, transcranial magnetic stimulation, variability, compartmental cable equation

(Some figures may appear in colour only in the online journal)

\section{Introduction}

During resective surgery, for example when removing a tumour or an epileptogenic area, the patient may risk impairment of his functions (Pouratian and Bookheimer 2010). Therefore, intraoperative functional mapping by direct cortical stimulation (DCS) is commonly used. When operating in the vicinity of motor or speech-related areas awake craniotomies are performed (Ojemann et al 1988, Corina et al 2010). This mapping is the gold standard, however, preoperative evaluation with transcranial magnetic stimulation (TMS) can be of great value (Lioumis et al 2012, Krieg et al 2014). TMS is a non-invasive technique that can modulate brain activity. When delivered over a specific cortical site, it can create a 'virtual lesion' and temporarily disrupt the ongoing process in that stimulated area. It may help in objective riskbenefit balancing of the planned surgery, and it may allow more targeted, smaller craniotomies, and also faster and safer intraoperative mapping (Picht et al 2013). In addition for a patient that cannot undergo awake craniotomy, it may lead to safer surgery. It has been shown that navigated TMS can localise the cortical motor representations as accurately as DCS (Picht et al 2011) and more accurately than functional magnetic resonance imaging (fMRI) (Forster et al 2011) in tumour patients. Despite the good overall correlation between the speech-related regions identified with navigated TMS and DCS, low specificity in posterior language areas is observed (Picht et al 2013). Where the motor skills are organised clearly and specifically in the primary motor cortex and therefore relatively easy to localise (Penfield and Rasmussen 1950), language is derived from an extended, complex and highly individualised neural network of cortical and subcortical connections (Catani et al 2005).

TMS has been used to disturb speech performance during naming tasks. In contrast to TMS over the primary motor cortex, which is a small targeted area, speech mapping experiments reveal a large spread of the targeted cortical sites that lead to naming errors. Several speech mapping studies have demonstrated the high variability in number, type and location of the naming errors among their subjects (Lioumis et al 2012, Picht et al 2013, Hernandez-Pavon et al 2014). Besides this inter-subject variability, speech cortical mapping by TMS shows also to be variable within single subjects. In the studies (Lioumis et al 2012) and (Picht et al 2013), the left hemisphere was stimulated on average at 360 and 450 sites of which 13 and 61 led to a naming error, respectively. Small perturbations in the stimulated sites made the difference between a disturbed or normal speech performance. Moreover, Lioumis et al (2012) retested the sites where errors occurred and indicated that most but not all of them were reproducible and not always of the same type. In this paper, we aim to numerically explain the observed variability of TMS responses during a speech mapping experiment.

For this purpose, we apply the in-house developed computational model for TMS (De Geeter et al 2011, 2012, De Geeter et al 2016). This model estimates the electric fields that are induced in the brain by TMS and their impact on neuronal structures, such as white matter fibres. Realistic 3D reconstructions of bundles of grouped white matter fibres, called 
tracts, can be generated using tractography based on the diffusion tensor imaging (DTI) data of the subject's brain (Basser et al 2000). The inclusion of white matter fibre trajectories with DTI-based tractography as a priori information is a more realistic approach than in most TMS studies published so far. Moreover, the combination of electromagnetic field modelling with models describing neurophysiology is innovative. The variation of the membrane potentials along the targeted tracts due to the induced electric field and consequently the possible generation of action potentials is calculated to study the electrical neuronal activity.

The applied DTI-based computational model consists of the following steps. First, a personalized head model is constructed with realistic geometry and tissue anisotropy using MRI. The position and orientation of the TMS coil relative to the head are directly retrieved from the neuronavigation system. The induced electric field distribution is then calculated in the whole brain for each specific case using the anisotropic independent impedance method (De Geeter et al 2012). This method has been validated by comparison with analytical and standard finite element methods (De Geeter et al 2011). Close to the targeted region of interest, relevant tracts are reconstructed using DTI-based tractography. The electric field along these tracts affects the neural membrane potentials. These membrane potentials as function of time and space are computed using a compartmental cable equation (Salvador 2009, De Geeter et al 2016), which is based on the neurophysiology and in accordance with the Hodgkin-Huxley dynamics. Salvador (2009) compared his results obtained with the compartmental cable equation with those obtained with NEURON (http://neuron.yale.edu/neuron), a program that is commonly used in the scientific literature for the calculation of the neuron's response, and consequently validated the method. By combining the induced electric fields with the neuronal responses, this computational model accounts for spatial and temporal effects of TMS in the brain. Moreover, the use of MRI, tractography and navigated TMS allows to treat each subject or patient and each case individually.

\section{Methodology}

\subsection{Speech mapping case study}

The speech mapping experiment was performed on a healthy, right-handed, 41 year old male subject at the Helsinki University Hospital, Finland, following Lioumis' approach (Lioumis et al 2012). The subject gave informed consent to participate in this study under a protocol approved by the ethical committee of the Helsinki University Hospital. During the first session, the baseline performance was measured while the subject was naming normalized pictures of simple objects (Brodeur et al 2010) as quickly and precisely as possible under normal conditions. The images were placed in random order. Those that were unfamiliar or named incorrectly were removed from the set. During the second session, the subject named these images again, while repetitive TMS was delivered to semi-random left-hemispheric areas. Traditionally, the language centres are situated in the left hemisphere of right-handed individuals. The areas were chosen semi randomly, since a priori anatomical information about the language related areas was used and the coil was moved around the hemisphere but returned more often to those site where a naming error occurred. The eXimia navigated brain stimulation (Nexstim Ltd., Helsinki, Finland) was used in combination with a biphasic $70 \mathrm{~mm}$ figureof-eight coil. The neuronavigation system monitored the coil position and orientation relative to the subject's head during this speech mapping experiment. Every $2.5 \mathrm{~s}$ an object image was presented for $0.7 \mathrm{~s}$ on the computer screen. During the second session, navigated TMS was applied $0.3 \mathrm{~s}$ after the image presentation onset, similar to Lioumis et al (2012). 5 pulses, with a pulse width of $230 \mu \mathrm{s}$ and an intensity of $45 \%$ of the motor threshold, were delivered at $5 \mathrm{~Hz}$ 
spread over a total duration of $1 \mathrm{~s}$. The performance of the subject, together with the TMS coil and object images were video-recorded, as in Lioumis et al (2012), so to analyse the results offline. The baseline responses were compared with those during TMS. A neurophysiologist categorised the naming errors induced by TMS by type and location. The different types are no-response error (anomic aphasia or anomia), phonologic paraphasia, semantic paraphasia and performance errors. On a total number of 263 object images presented, 15 naming errors occurred.

We selected four cases in which the coil was moved, rotated and tilted with limited deviations. One might assume that almost the same stimulation is performed. However, in one case, denoted as E, a naming error (more specifically a performance error) occurred, while in the other three cases, denoted as $\mathrm{NE}_{\mathrm{A}}, \mathrm{NE}_{\mathrm{B}}$ and $\mathrm{NE}_{\mathrm{C}}$, the subject appointed the object images as smoothly as without TMS.

\subsection{Realistic personalized head model}

A T1-weighted image, with voxelsize $1 \times 1 \times 1 \mathrm{~mm}^{3}$, and diffusion-weighted images (DTI), with voxelsize $1 \times 1 \times 5 \mathrm{~mm}^{3}, 25$ diffusion directions and a $b$-value of $1000 \mathrm{~s} \mathrm{~mm}^{-2}$, were acquired from the subject on a GE Signa HDxt 1.5T MRI scanner at the Helsinki University Hospital, Finland. These data sets were post-processed using statistical parametric mapping (SPM12) (Friston et al 2007) for the construction of a realistic $2 \mathrm{~mm}$ resolution anisotropic head model. This $112 \times 128 \times 100$ head model was segmented in the tissues scalp, skull, cerebrospinal fluid (CSF), grey and white matter. Their electrical properties were obtained from the frequency-dependent 4-Cole-Cole model (Cole and Cole 1941, Gabriel et al 1996) and the subject-specific DTI. The isotropic conductivity values of the considered tissues that correspond to the fundamental stimulation frequency $(4.348 \mathrm{kHz})$ of the applied excitation current in the TMS device are 0.0012, 0.0203, 2.0, 0.1083 and $0.0662 \mathrm{~S} \mathrm{~m}^{-1}$, respectively. Anisotropic conductivity tensors were computed from the diffusion tensors, as explained in De Geeter et al (2012).

\subsection{Electromagnetic modelling}

The induced electric field distributions were computed for the four cases using the anisotropic independent impedance method (De Geeter et al 2011, 2012). Based on these distributions, the centre of the region of interest (ROI) was defined. This is sometimes done by searching the overall maximum electric field value. Another possible method is to search the first voxel in cerebral tissue which is situated in the extension of the coil normal vector. In this study, we have chosen the ROI centre by calculating the position of the weighted mean. Within a sphere of radius $6 \mathrm{~cm}$ around the coil centre, the voxel positions are weighted by their electric field value. Finally, a $10 \times 10 \times 10 \mathrm{~mm}^{3}$ ROI box was created centralized around this ROI centre. Whole brain tractography (Basser et al 2000) is performed on the $1 \mathrm{~mm}$ DTI using the graphical toolbox ExploreDTI (Leemans et al 2009). The step size is set to $1 \mathrm{~mm}$, the minimum fibre length to $50 \mathrm{~mm}$, the fractional anisotropy (FA) threshold to 0.2 and the bending angle threshold to 30 degrees. Although there is a user-defined influence, these main tractography parameters are in line with the conventional settings range in current literature. This way, realistic 3D trajectories of bundles of grouped white matter fibres, also called tracts, are reconstructed and those that traverse the seed ROI box are identified. In order to investigate the location and functional meaning of these tracts in terms of the speech-related network, we use the automated anatomical labeling (AAL) atlas (Tzourio-Mazoyer et al 2002). This atlas divides a spatially normalized single-subject high-resolution $(1 \mathrm{~mm})$ brain in anatomical 
volumes of interest (AVOIs), namely 45 AVOIs for each hemisphere of the cerebrum and 26 for the cerebellum. SPM12 (Friston et al 2007) is applied for the coregistration of the subject's head model with the AAL atlas.

\subsection{Neurophysiologic modelling}

When applying an electric field on neurons, their membrane potential is altered (Roth and Basser 1990). If this change is significant, action potentials might be initiated and propagated along the tracts. This response is described by a compartmental cable equation (Nagarajan et al 1993, Salvador 2009, De Geeter et al 2016). The induced electric field $E$ is mapped along the neural trajectories $l$ of the considered tracts and serves as the input for stimulation. Indeed, the directional derivative of this $E_{l}$ along the neural fibre describes the effect of stimulation by electromagnetic induction, such as TMS, on the membrane potential $V$. All tracts are assumed to be myelinated neurons, containing compartments representing passive dendrites, a passive soma, an active axon hillock, an active initial segment and alternating passive myelinated internodes and active Ranvier nodes, and are modelled with passive and active membrane properties. Their behaviour as function of time and space is computed by the following compartmental cable equation (De Geeter et al 2016):

$$
\begin{aligned}
c_{\mathrm{m}} \frac{\partial V(l)}{\partial t}+i_{\text {ion }}(l)= & \frac{1}{\Delta l^{2} r_{\mathrm{a}}}(V(l-\Delta l)-2 V(l)+V(l+\Delta l)) \\
& +\frac{1}{\Delta l r_{\mathrm{a}}}\left(E_{l}(l-\Delta l)-E_{l}(l)\right) .
\end{aligned}
$$

$c_{\mathrm{m}}$ is the membrane's capacitance per unit length and $r_{\mathrm{a}}$ the axial resistance per unit length at the intracellular space. For the passive components, the ionic current per unit length equals

$$
i_{\text {ion }}(l)=g_{\mathrm{m}}\left(V(l)-V_{\mathrm{r}}\right)
$$

whereas it describes a more complex behaviour for the active components

$$
\begin{aligned}
i_{\text {ion }}(l)= & g_{\mathrm{Na}} m^{3} h\left(V(l)-V_{\mathrm{Na}}\right)+g_{\mathrm{K}} n^{4}\left(V(l)-V_{\mathrm{K}}\right) \\
& +g_{\mathrm{L}}\left(V(l)-V_{\mathrm{L}}\right) .
\end{aligned}
$$

$V_{\mathrm{r}}$ is the resting membrane potential and $V_{c h}(c h=\mathrm{Na}, \mathrm{K}, \mathrm{L})$ the Nernst potentials of the sodium, potassium and leakage channel, respectively. The factors $m^{3} h$ and $n^{4}$ represent the probabilities that the sodium and potassium channels are open with a conductance per unit length of $g_{\mathrm{Na}}$ and $g_{\mathrm{K}}$ and are voltage and time dependent. For the equations of the transition rates, we refer to De Geeter et al (2016). $g_{\mathrm{L}}$ is the leak conductance per unit length and $g_{\mathrm{m}}=1 / r_{\mathrm{m}}$ the conductance per unit length related to the membrane resistance. The applied parameter values are based on human sensory fibre data (Wesselink et al 1999). The Crank-Nicolson method is used to discretise equation (1). Since there is a difference of several orders of magnitude between the lengths of the various compartments, we implement a variable space step $\Delta l$, as in De Geeter et al (2016). Finally, the spatio-temporal variation of the membrane potentials along all considered white matter fibre tracts is computed for the four cases.

\section{Results and discussion}

As mentioned before, four cases with very small perturbations in coil position and orientation were selected. In case $\mathrm{E}$ a naming error occurred, while in the other cases $\left(\mathrm{NE}_{\mathrm{A}, \mathrm{B}, \mathrm{C}}\right)$ the subject appointed the images as smoothly as without TMS. From the neuronavigation system 
Table 1. Coil specifications of the four selected study cases (neurological convention).

\begin{tabular}{llll}
\hline Coil & Position $\mathbf{O}$ & Normal $\mathbf{n}$ & Direction $\mathbf{~}$ \\
\hline $\mathrm{E}$ & $(14.08,76.28,45.02)$ & {$[-0.972,-0.043,0.230]$} & {$[-0.066,0.994,-0.091]$} \\
$\mathrm{NE}_{\mathrm{A}}$ & $(15.16,79.21,44.73)$ & {$[-0.967,0.024,0.253]$} & {$[0.016,0.999,-0.037]$} \\
$\mathrm{NE}_{\mathrm{B}}$ & $(14.36,77.00,44.64)$ & {$[-0.975,-0.051,0.215]$} & {$[-0.067,0.995,-0.070]$} \\
$\mathrm{NE}_{\mathrm{C}}$ & $(13.55,76.24,45.42)$ & {$[-0.974,-0.102,0.203]$} & {$[-0.115,0.992,-0.054]$} \\
\hline
\end{tabular}
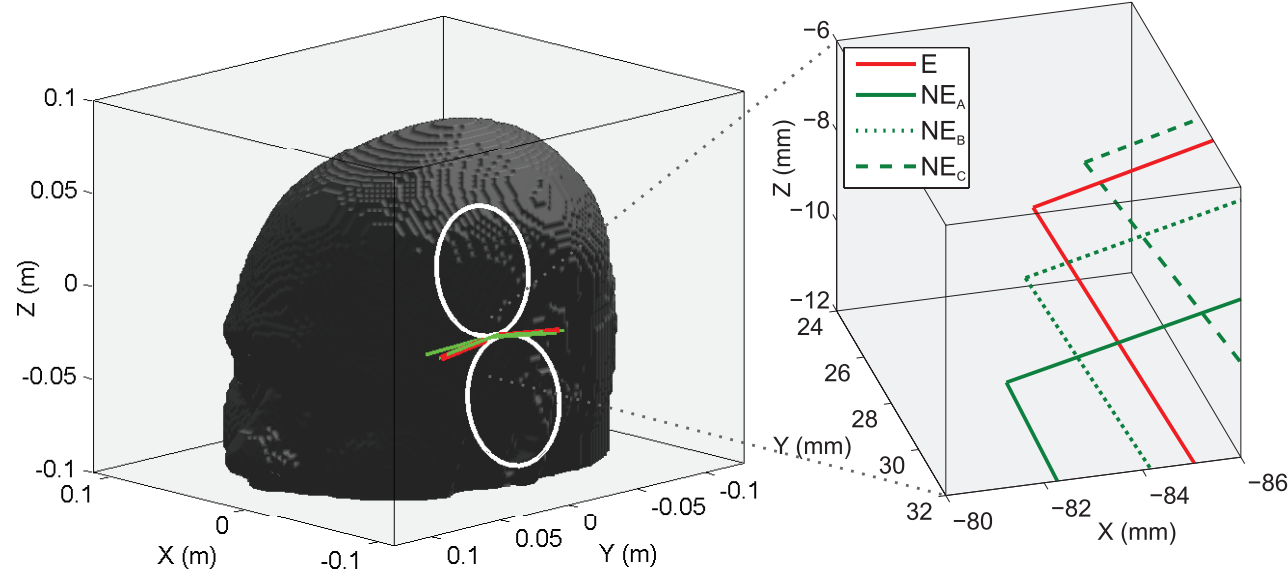

Figure 1. (Left) The coil position with respect to the head model. The red and green lines represent the coil normal $\mathbf{n}$ and the induced current direction $\mathbf{m}$ for the cases that did $(\mathrm{E})$ and did not $\left(\mathrm{NE}_{\mathrm{A}}, \mathrm{NE}_{\mathrm{B}}, \mathrm{NE}_{\mathrm{C}}\right)$ provoke a naming error, respectively. The outer windings of the $70 \mathrm{~mm}$ figure-of-eight biphasic Nexstim coil are visualised for the error case. (Right) Zoom in of the small perturbations in coil position and orientation. Coordinate $(0,0,0)$ refers to the centre of the head model volume.

we retrieved all needed coil configuration parameters of these four cases, i.e. the coordinates $\mathbf{O}$ of the centre of the coil, the unit vector $\mathbf{n}$ normal to the plane of the coil and directed away from the subject's head, and the unit vector $\mathbf{m}$ in the direction of maximal induced currents (in homogeneous material). They are listed in table 1.

The nasion, and the right and left pre-auricular points of the subject were denoted by a digitizing pen at the beginning of the TMS experiment and their positions were saved by the navigation system. By using the coordinates of these landmarks, the coil specifications were co-registered to the head model. The values of table 1 are already expressed according to the voxel sequence of this head model. Voxel $(0,0,0)$ refers to the coordinates of the lower left hand rear corner of the $112 \times 128 \times 100$ head model volume. The coordinate system $(x, y, z)$ is defined according to the neurological convention. The $x$-axis is oriented from left to right, the $y$-axis from posterior to anterior and the $z$-axis from inferior to superior. To calculate the coordinates in meters relative to the centre, one must subtract $(56,64,50)$ from it and multiply the result by the voxelsize of $2 \mathrm{~mm}$. Figure 1 illustrates for the four cases how the coil is positioned and oriented relative to the head model. The two vectors $\mathbf{n}$ and $\mathbf{m}$ for the naming error case are indicated in red and for the non-error cases in green. The origin of these vectors are located in coordinate $\mathbf{O}$ of the centre of the coil. For example, for case $\mathrm{E}$ the origin of the vectors corresponds to voxel $(14.08,76.28,45.02)$ and thus coordinates $(-0.084,0.025$, $-0.010)$ in $\mathrm{m}$. 


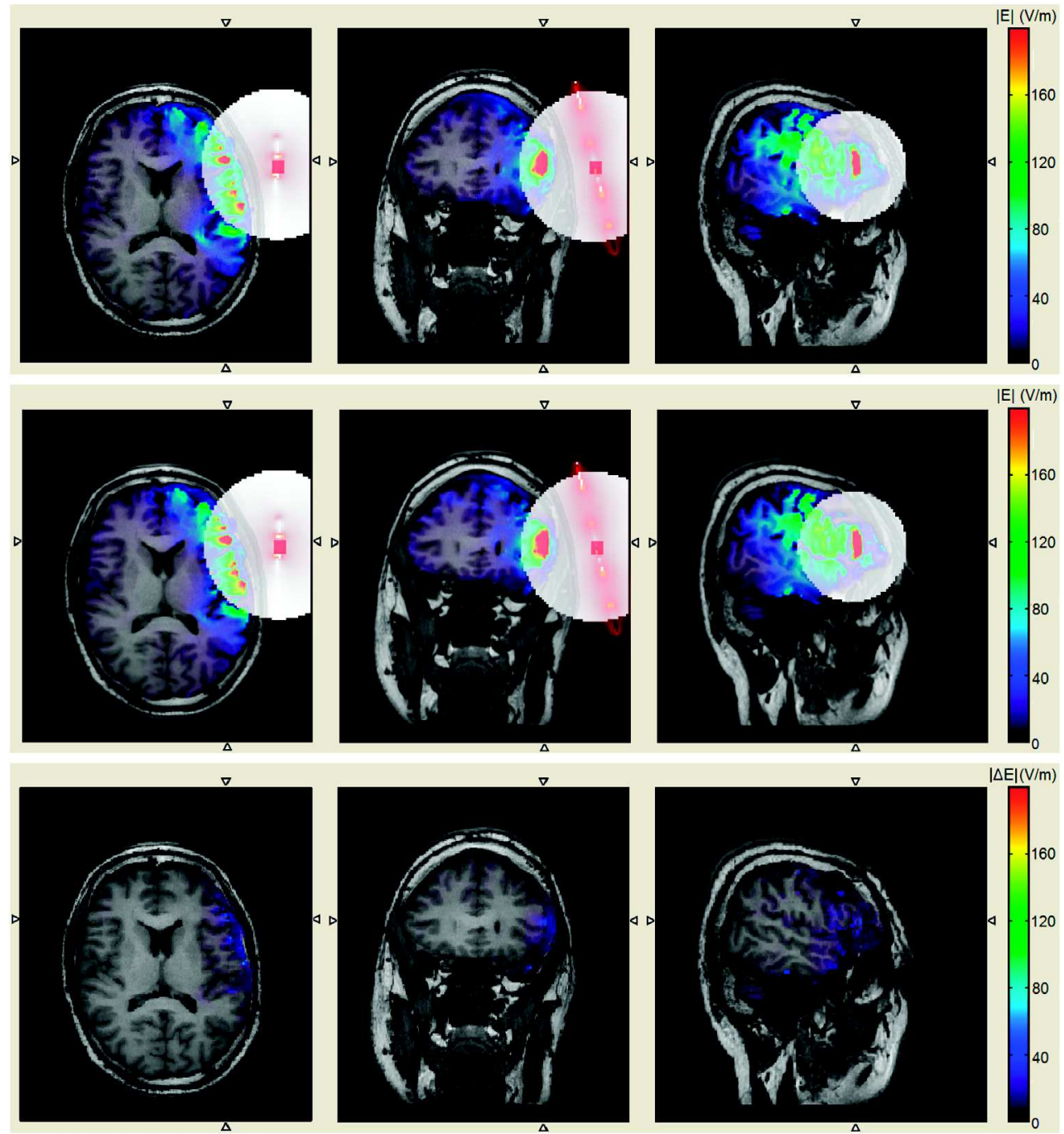

Figure 2. (Left) Axial, (middle) coronal and (right) sagittal view of the induced electric field amplitude distribution due to (above) the E stimulus and (middle) the $\mathrm{NE}_{\mathrm{A}}$ stimulus. (Below) Absolute difference map of the electric field distributions. Only the values in the cerebral tissues are visualised for clarity reasons. The white mask indicates the sphere of radius $6 \mathrm{~cm}$ around the coil centre. The original T1-weighted image is visualised on the background.

The electric field distributions induced in the brain for cases $\mathrm{E}$ and $\mathrm{NE}_{\mathrm{A}}$ and their absolute difference are shown in figure 2. At first sight, only minor differences can be noticed. Therefore, the distributions for the other two cases are not presented. Table 2 lists all possible ROI centres, calculated by the three different methods based on the induced electric field distributions. It indicates that the overall maximum electric field values (pos max) are located in the same voxel for the four cases. Since the normal vectors are slightly different, see table 1, the first cerebral voxels situated in the extension of the coil normal vector (pos normal) are scattered up to $5 \mathrm{~mm}(1$ voxel $=2 \mathrm{~mm}$ ) from each other. The last selection method, chosen in this study, uses the positions of the weighted mean (pos weighted). They are shown in 
Table 2. Selection methods for the seed ROI centre (neurological convention).

\begin{tabular}{llll}
\hline Coil & Pos max & Pos normal & Pos weighted \\
\hline $\mathrm{E}$ & $(34,80,45)$ & $(30,77,42)$ & $(33,71,46)$ \\
$\mathrm{NE}_{\mathrm{A}}$ & $(34,80,45)$ & $(31,79,41)$ & $(34,74,46)$ \\
$\mathrm{NE}_{\mathrm{B}}$ & $(34,80,45)$ & $(31,78,42)$ & $(33,71,45)$ \\
$\mathrm{NE}_{\mathrm{C}}$ & $(34,80,45)$ & $(30,78,43)$ & $(32,71,46)$ \\
\hline
\end{tabular}
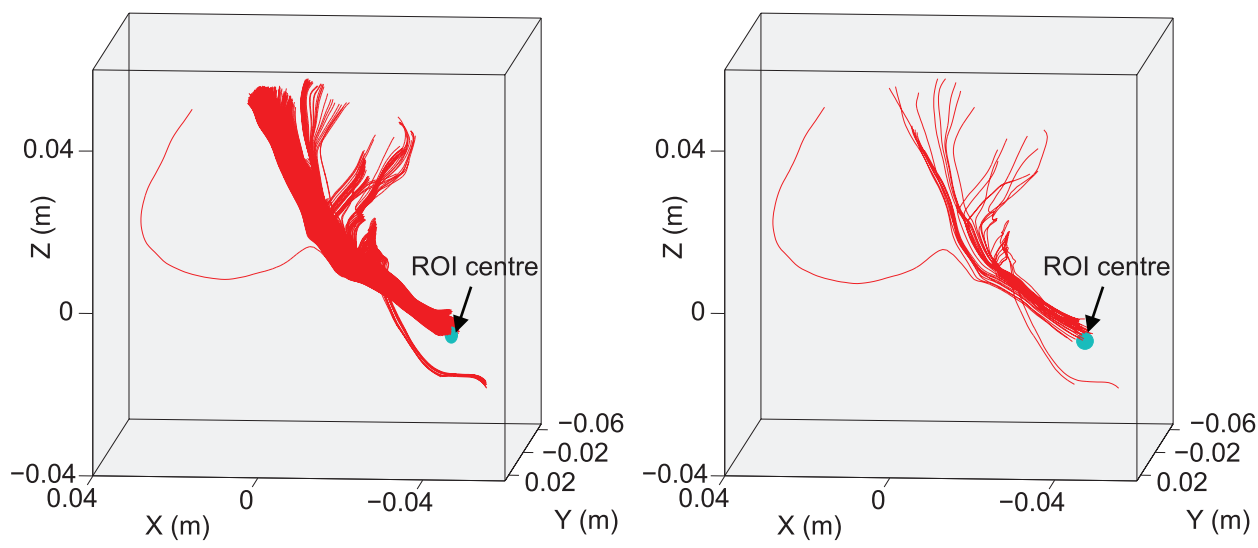

Figure 3. Reduction of the 1239 white matter fibre tracts (left) to the 35 selected tracts (right), represented by red lines. The seed ROI centre is depicted by a blue dot.

the last column of table 2. Note that the positions are again expressed according to the voxel sequence. We defined voxel $(33,71,46)$ as the seed ROI centre, corresponding to coordinates $(-0.046,0.014,-0.008)$ in $\mathrm{m}$. Within its $5 \mathrm{~mm}$ range, the ROI box is created. From the extensive whole brain tractogram, the white matter fibre tracts that traverse this seed ROI box are extracted. Moreover, fibre tracts that are closely related, i.e. both their first and last points are located less than $5 \mathrm{~mm}$ from another, are considered as the same bundle. This additional selection criterion reduced the number of relevant tracts from 1239 to 35 , see figure 3 .

The behaviour of the membrane potentials in space and time for case $\mathrm{NE}_{\mathrm{A}}$ is shown in figure 4. A detail of this spatio-temporal variation is presented within the tract with label number 21 to facilitate interpretation. The TMS pulse was applied during the first $230 \mu$ s and caused the membrane potential to increase from its resting value $(-84 \mathrm{mV})$ to values up to $40 \mathrm{mV}$. This depolarization resulted in the initiation of an action potential that propagated unattenuated along the fibre tract till it reached the end. We consider this sequence of events as electrical activity in the white matter fibre tract. Figure 4 illustrates thus clearly that 13 out of the 35 tracts are activated for case $\mathrm{NE}_{\mathrm{A}}$.

The blue-coloured tracts in figure 5 show where activity is generated by the TMS pulse for all cases and reveal a large variability. One tract, more specifically tract 21 , is activated for all coil positions. Another tract, tract 33, is only triggered for E, the case where a naming error is elicited. $\mathrm{NE}_{\mathrm{A}}$ induced action potentials in a total of 13 tracts, whereas $\mathrm{NE}_{\mathrm{B}}$ stimulated 11 tracts and $\mathrm{NE}_{\mathrm{C}}$ only one.

To interpret the location and functional meaning of these tracts in terms of the speechrelated network, we use the anatomical volumes of interest of the AAL atlas. Table 3 lists the AAL AVOIs (Tzourio-Mazoyer et al 2002) being closest to the start and end points of the 35 considered tracts, together with the smallest distances (dist) between the coordinates of 


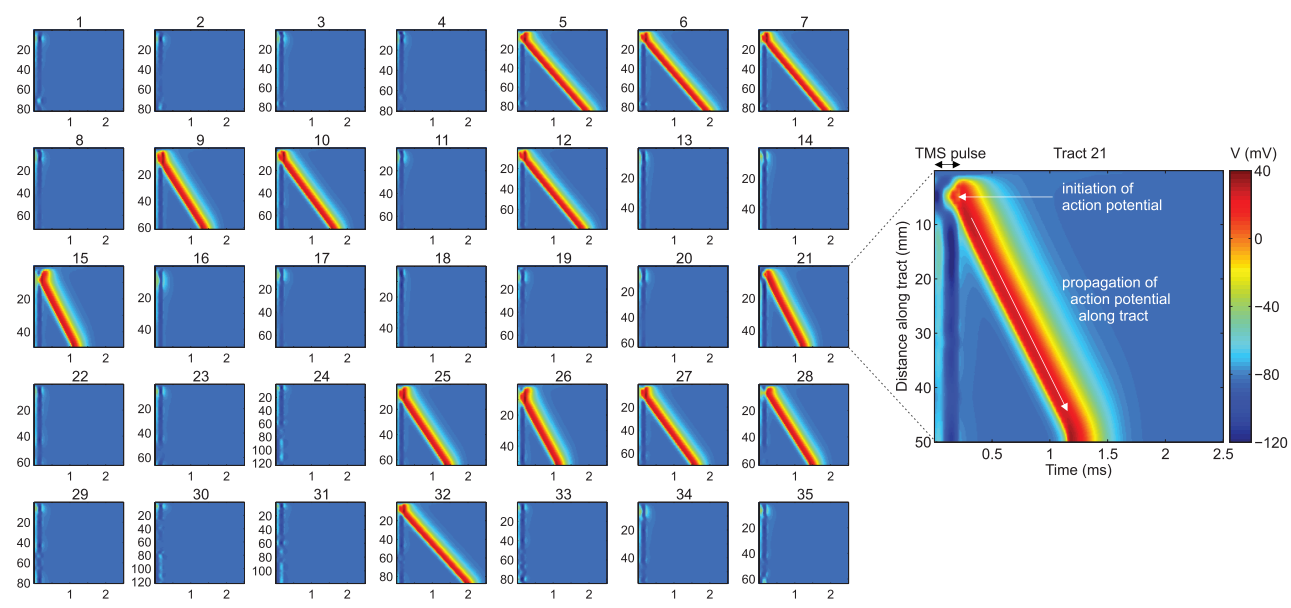

Figure 4. (Left) The spatio-temporal variations of the membrane potential $V$ within all 35 considered fibre tracts for case $\mathrm{NE}_{\mathrm{A}}$. (Right) Detail for tract 21 . The distances along the tracts are measured starting from the point closest to the ROI centre. Note that these distances vary among the different tracts, depending on their total length.

these points and the centre of the nearest AVOIs. Those tracts which are activated due to the stimulus in one or more cases are indicated in italics.

Since the seed ROI box was centralised around voxel $(33,71,46)$, corresponding to the opercular part of the left inferior frontal gyrus (Front Inf Oper L), it is logical to explain that all considered tracts have their start point lying nearby this AAL AVOI. The opercular part of the left inferior frontal gyrus contains Broca's area, which is responsible for speech production (Catani et al 2005, Orrison 2008). Tract 21, activated in all cases, connects the left inferior frontal gyrus with the left Heschl gyrus (Heschl L), also known as the transverse temporal gyrus. It is part of the temporal lobe and contains the primary auditory cortex (Brodmann area BA41) (Brodmann 1909). Tract 33, which is only triggered for the error case, is connected to the left postcentral gyrus (Postcentral L), also known as the primary somatosensory cortex (S1), and the left inferior parietal lobe (Parietal Inf L). The inferior parietal lobe is involved with sensorimotor integration, spatial attention and auditory processing. It contains Wernicke's area, where language semantics are processed (Brodal 2004, Catani et al 2005). Note that tracts 27 and 32, which also connect the opercular part of the left inferior frontal gyrus with the left inferior parietal lobe, are activated for cases $\mathrm{NE}_{\mathrm{A}}$ and $\mathrm{NE}_{\mathrm{B}}$ without eliciting a naming error. Both tracts have slightly different trajectories, which can be seen from figure 5 and table 3 . For example, the start point of tract 33 lies at a distance of $10.00 \mathrm{~mm}$ to its nearest AVOI against 14.54 and $14.61 \mathrm{~mm}$ for tracts 27 and 32, respectively. $10.00 \mathrm{~mm}$ is relatively short compared to the mean distance of $14.51 \mathrm{~mm}$ with standard deviation $4.29 \mathrm{~mm}$. When investigating the spatio-temporal distribution of the membrane potential within tract 33 for coil position E, see figure 6, a more important difference is detected. It is the only case in which the action potential originates at the end point and is conducted towards the start point of the tract, located closest to the ROI. The orientation of tract 33 can be identified from figure 5 by comparing case $\mathrm{E}$ (where tracts 21 and 33 are coloured blue) with case $\mathrm{NE}_{\mathrm{C}}$ (where only tract 21 is coloured blue). This orientation with respect to the TMS coil determines the stimulation mechanism, i.e. the directional derivative of the induced electric field mapped along the neural fibre trajectory $\left(\frac{E_{l}(l-\Delta l)-E_{l}(l)}{\Delta l}\right)$. As shown in figure 6, for tract 33 this term has larger values at the end points than at the start points, resulting in depolarization of the 

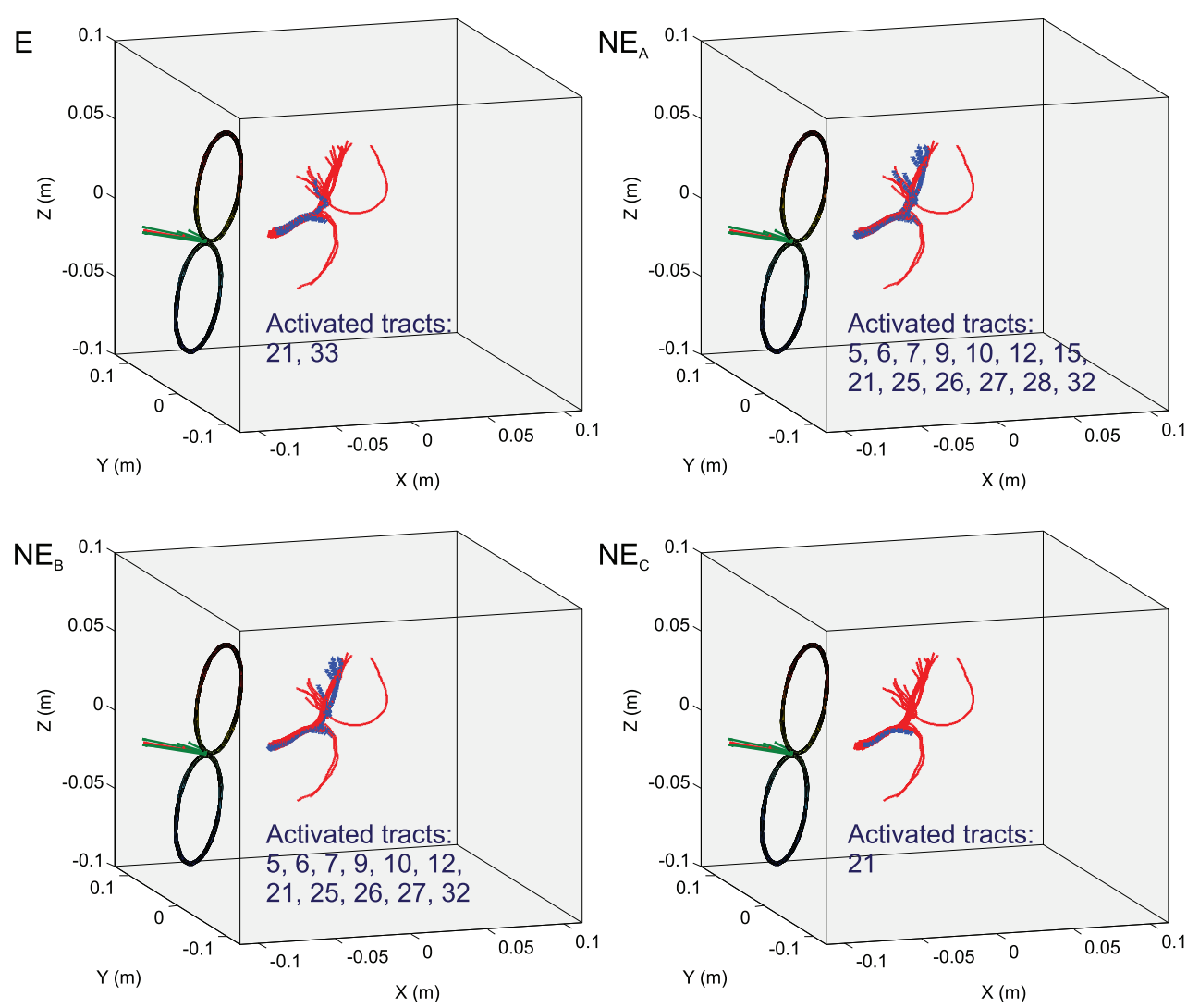

Figure 5. The 35 selected white matter fibre tracts with respect to the coil position, similar as figures 1 and 3 , for cases $\mathrm{E}, \mathrm{NE}_{\mathrm{A}}, \mathrm{NE}_{\mathrm{B}}$ and $\mathrm{NE}_{\mathrm{C}}$. The tracts which are activated, i.e. when an action potential is conducted along them due to the stimulus, are coloured blue instead of red.

membrane potential at the end points and thus the obtained reversed direction of action potential. Error case E can thus be distinguished from the other cases by sending electrical activity along tract 33 from Wernicke to Broca's area.

The other relevant AVOIs are the left precentral gyrus (precentral L), the right and left supplementary motor area (SMA R and L) and the left middle temporal gyrus (temp mid L). The precentral gyrus contains the primary motor cortex (M1). Besides M1, the motor cortex consists of the premotor cortex and the supplementary motor area (SMA), together located in BA6. These motor area are mainly involved in the planning, control and execution of movements such as of the muscles used to pronounce words. The exact function of the middle temporal gyrus (BA21) is unclear, but it has been connected with auditory processing and accessing word meaning while reading (Brodmann 1909, Brodal 2004, Orrison 2008).

The variability of the TMS effects is reported in many clinical and psychological studies (Lioumis et al 2009, Casarotto et al 2010, Hernandez-Pavon et al 2014). Different outcomes among subjects might be ascribed to the inter-subject variability of brain size and morphology. Amunts et al (1999) studied for example Broca's area in ten post-mortem human brains and revealed significant variations in size as well as in the relation of this area to sulcal landmarks. However, a high intra-subject variability of the TMS effects is also observed, 
Table 3. AAL AVOIs connected by the tracts.

\begin{tabular}{|c|c|c|c|c|}
\hline \multirow{2}{*}{$\begin{array}{l}\text { Tract } \\
\mathrm{nr}\end{array}$} & \multicolumn{2}{|c|}{ Start point } & \multicolumn{2}{|c|}{ End point } \\
\hline & AAL AVOI & Dist (mm) & AAL AVOI & Dist (mm) \\
\hline 1 & Front Inf Oper L & 14.26 & SMA L & 6.94 \\
\hline 2 & Front Inf Oper L & 14.05 & SMA L & 9.48 \\
\hline 3 & Front Inf Oper L & 15.08 & SMA L & 18.88 \\
\hline 4 & Front Inf Oper L & 14.38 & SMA L & 11.03 \\
\hline 5 & Front Inf Oper L & 15.56 & $S M A L$ & 19.94 \\
\hline 6 & Front Inf Oper L & 15.33 & $S M A L$ & 20.74 \\
\hline \multirow[t]{2}{*}{7} & Front Inf Oper L & 15.95 & $S M A L$ & 21.07 \\
\hline & Front Inf Oper L & 13.54 & SMA L & 10.05 \\
\hline 9 & Front Inf Oper L & 15.45 & Postcentral L & 21.66 \\
\hline \multirow[t]{2}{*}{10} & Front Inf Oper L & 15.19 & Precentral L & 7.20 \\
\hline & & & Postcentral L & 15.37 \\
\hline 11 & Front Inf Oper L & 13.35 & SMA L & 10.58 \\
\hline 12 & Front Inf Oper L & 14.83 & Precentral L & 16.34 \\
\hline 13 & Front Inf Oper L & 12.46 & Heschl L & 21.82 \\
\hline 14 & Front Inf Oper L & 12.54 & Heschl L & 21.52 \\
\hline 15 & Front Inf Oper L & 13.94 & Precentral L & 22.47 \\
\hline 16 & Front Inf Oper L & 14.26 & Precentral L & 23.63 \\
\hline 17 & Front Inf Oper L & 12.60 & SMA L & 10.83 \\
\hline 18 & Front Inf Oper L & 11.16 & Heschl L & 21.59 \\
\hline 19 & Front Inf Oper L & 13.44 & SMA L & 11.29 \\
\hline 20 & Front Inf Oper L & 12.00 & SMA L & 15.91 \\
\hline 21 & Front Inf Oper L & 12.45 & Heschl L & 20.75 \\
\hline \multirow[t]{2}{*}{22} & Front Inf Oper L & 10.33 & Precentral L & 6.53 \\
\hline & & & Postcentral L & 13.66 \\
\hline 23 & Front Inf Oper L & 10.44 & Precentral L & 16.57 \\
\hline 24 & Front Inf Oper L & 10.51 & Temp Mid L & 18.35 \\
\hline 25 & Front Inf Oper L & 14.70 & Postcentral L & 22.35 \\
\hline 26 & Front Inf Oper L & 14.08 & Precentral L & 16.55 \\
\hline 27 & Front Inf Oper L & 14.54 & Parietal Inf L & 25.02 \\
\hline 28 & Front Inf Oper L & 10.96 & Precentral L & 17.59 \\
\hline \multirow{2}{*}{29} & Front Inf Oper L & 10.75 & Postcentral L & 8.76 \\
\hline & & & Parietal Inf L & 15.68 \\
\hline \multirow[t]{2}{*}{30} & Front Inf Oper L & 12.99 & SMA R & 13.74 \\
\hline & & & SMA L & 15.03 \\
\hline 31 & Front Inf Oper L & 10.14 & Temp Mid L & 14.90 \\
\hline \multirow[t]{2}{*}{32} & Front Inf Oper L & 14.61 & Postcentral L & 11.31 \\
\hline & & & Parietal Inf L & 16.94 \\
\hline \multirow[t]{2}{*}{33} & Front Inf Oper L & 10.00 & Postcentral L & 9.99 \\
\hline & & & Parietal Inf L & 18.40 \\
\hline 34 & Front Inf Oper L & 10.63 & Precentral L & 16.64 \\
\hline 35 & Front Inf Oper L & 10.40 & Precentral L & 16.68 \\
\hline
\end{tabular}

such as in this speech mapping study. Whether or not a naming error was elicited appeared to be due to small perturbations in coil position and orientation, see table 1 and figure 1 . The calculated results, summarized in figure 5 , are certainly sensitive to the coil specifications. In this way, we obtained similar variability in the simulations as in the experiment for the considered case study. 

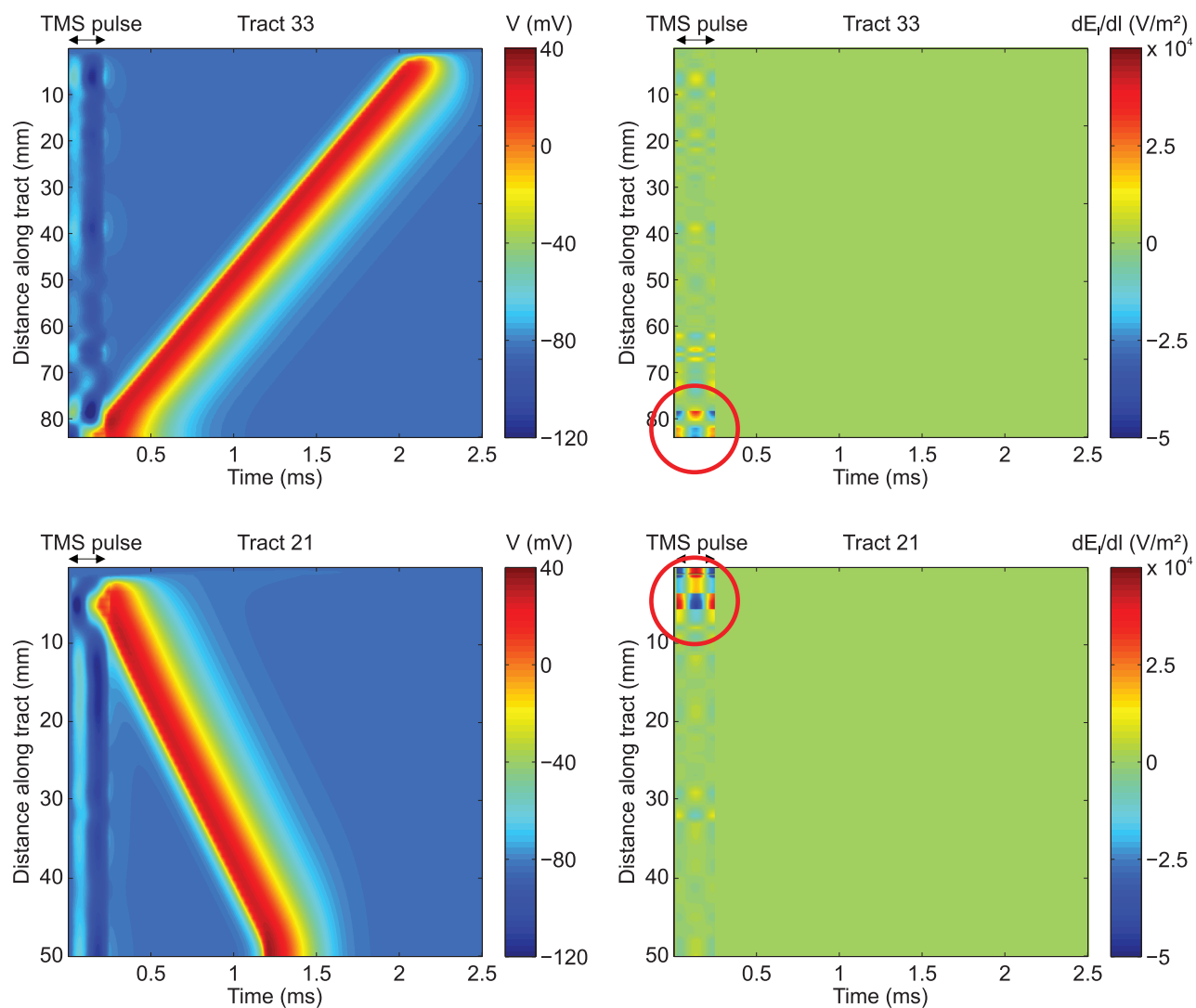

Figure 6. (Above) The spatio-temporal variations of the membrane potential $V$ (left) within tract 33 for case $\mathrm{E}$ and its triggering factor, the directional derivative of the induced electric field mapped along the neural fibre trajectory $\frac{\mathrm{d} E_{l}}{\mathrm{~d} l}$ (right). The distance along the tract is measured starting from the point closest to the ROI centre. (Below) As a reference, similar plots for tract 21 , case $\mathrm{E}$.

We are aware of the fact that our model still has uncertainties. The 4-Cole-Cole model should be used with caution to calculate conductivity values at low frequencies, such as the applied $4.348 \mathrm{kHz}$ (Cole and Cole 1941, Gabriel et al 1996). Though, cerebral voxels have shown limited sensitivity of the induced electric field to changes in conductivity (De Geeter et al 2012). The geometrical and electrical neural property values, e.g. length and diameter, capacitance and conductance of the different neural compartments and the membrane's resting potential, vary among literature (Manola et al 2007). In a previous paper (De Geeter et al 2016) a sensitivity analysis was performed of the neural parameter values on the stimulation threshold and site. The results seemed to be highly sensitive to the initial state of the membrane potential and the intracellular resistivity, whereas they were less sensitive to changes in the lengths and diameters of the different compartmental segments. For the fixed chosen set of parameters, based on human sensory fibre data (Wesselink et al 1999), that we have applied in our calculations, variability is obtained. By defining the ROI centre as the position of the weighted mean of the induced electric fields, more information on the total field distribution is included in comparison to the maximum value or those in the extension of the coil's normal vector. Nevertheless, the effect of method choice is limited by the use of the $10 \times 10 \times 10 \mathrm{~mm}^{3}$ ROI box within which the tracts must pass. 
We could not conclusively justify why TMS for the case E did elicit a naming error and did not for the other three cases. Even though, one white matter fibre tract (tract 33 connecting Broca's with Wernicke's area, two main regions responsible for the process and execution of speech) appeared to be triggered only for the error case and conducting the action potential in the opposite direction. This observation should be further clarified by refining the neural model and its parameters, observing more tracts and including synapses and, consequently, the complete neural network of connections.

The compartmental neuron model, used for the simulation of the membrane potentials, segments the tracts in simple compartments which are represented by cylinders or truncated cones (De Geeter et al 2016). A more accurate representation of the neuronal geometry that includes multiple dendrites and the main features of the axonal embranchment is advised, together with a distinction between the different neuron types, each with their own electrical parameters. The reconstructed white matter fibre trajectories were derived with DTI-based tractography using a deterministic streamline approach. With this approach, the bulk-averaged tissue properties such as the dominant fibre orientation are taken in each voxel, but individual axons, neurons and synapses are not resolved. Furthermore, DTI is limited in regions of complex fibre configurations, such as kissing and crossing fibres (Tournier et al 2011). Remark the acquired DTI data has a slice thickness of $5 \mathrm{~mm}$ that is much bigger than the in-plane resolution of $1 \times 1 \mathrm{~mm}^{2}$. Since this may affect the accuracy of the reconstructed white matter fibre tracts, further studies should use a more isotropic acquisition. Along with synapse, the network interactions between the targeted and other brain regions should be included in future work. More specifically, the targeted fibre bundles are connected with a network of other tracts to which they communicate. Based on DTI, subject-specific connectivity matrices can be obtained with the graphical toolbox ExploreDTI (Leemans et al 2009), from which network properties such as efficiency, path length and connectivity degree can be derived, as in Caeyenberghs et al (2013).

In the future, we should explore more study cases. Other areas, stimulated during this speech mapping experiment, with very small perturbations in coil position and orientation that did or did not affect naming should be considered, as well as the same stimulation area for other healthy, right-handed subjects.

\section{Conclusions}

We numerically explored the observed variability of TMS responses during a speech mapping experiment by investigating four cases with slightly changed coil position and orientation. At first sight, only minor differences could be noticed between the induced electric field distributions. However, when computing the corresponding membrane potentials along the relevant white matter fibre tracts, different subsets of tracts were activated. This demonstrated the sensitivity of the results to the coil specifications. One specific tract, connecting the opercular part of the left inferior frontal gyrus (Broca's area) with the left inferior parietal lobe (Wernicke's area), was only triggered for the case where a naming error occurred. This speech mapping case study illustrates the feasibility of including the variability of TMS responses in simulations. It encourages further research on other study cases with larger subject groups and on refining the neural model with synapses and network connections.

The use of neuronavigated TMS, to retrieve the coil parameters, and MRI, to construct the realistic anisotropic head model with reconstructed neural trajectories near the region of interest, allows to treat each case and each subject individually. We believe such case- and subject-specific modelling that captures both electromagnetic and neurophysiologic phenomena triggered by TMS is necessary to investigate the variability in TMS experiments, certainly 
when the stimulation interacts with complex neural networks, such as the speech-related network, that can differ significantly from person to person. While this paper focussed on a speech mapping case study in which TMS is used for preoperative planning, gaining a better understanding of the variability of the TMS effect has much wider interest, and is likely to be as important in the research setting as it is in the clinical setting.

\section{Acknowledgment}

The authors gratefully acknowledge the financial support of the Agency for Innovation by Science and Technology in Flanders (IWT SB/101133), the Special Research Fund of Ghent University (BOF 13/24J/161) and the Fund of Scientific Research Flanders (FWO).

\section{References}

Amunts K, Schleicher A, Bürgel U, Mohlberg H, Uylings H and Zilles K 1999 Broca's region revisited: cytoarchitecture and intersubject variability J. Comp. Neurol. 412 319-41

Basser P, Pajevic S, Pierpaoli C, Duda J and Aldroubi A 2000 In vivo fiber tractography using DT-MRI data Magn. Reson. Med. 44 625-32

Brodal P 2004 The Central Nervous System: Structure and Function (Oxford: Oxford University Press)

Brodeur M, Dionne-Dostie E, Montreuil T and Lepage M 2010 The Bank of Standardized Stimuli (BOSS), a new set of 480 normative photos of objects to be used as visual stimuli in cognitive research PloS One 5 e10773

Brodmann K 1909 Vergleichende Lokalisationslehre der Großhirnrinde in ihren Prinzipien dargestellt auf Grund des Zellenbaues (Leipzig: Barth)

Caeyenberghs K, Leemans A, Leunissen I, Michiels K and Swinnen S 2013 Topological correlations of structural and functional networks in patients with traumatic brain injury Front. Hum. Neurosci. 7726

Casarotto S, Lauro L, Bellina V, Casali A, Rosanova M, Pigorini A, Defendi S, Mariotti M and Massimini M 2010 EEG responses to TMS are sensitive to changes in the perturbation parameters and repeatable over time PLoS One 5 e10281

Catani M, Jones D and Ffytche D 2005 Perisylvian language networks of the human brain Ann. Neurol. $578-16$

Cole K and Cole R 1941 Dispersion and absorption in dielectrics: alternating current characteristics J. Chem. Phys. $9341-51$

Corina D, Loudermilk B, Detwiler L, Martin R, Brinkley J and Ojemann G 2010 Analysis of naming errors during cortical stimulation mapping: implications for models of language representation Brain Lang. 115 101-12

De Geeter N, Crevecoeur G and Dupré L 2011 An efficient 3D eddy-current solver using an independent impedance method for transcranial magnetic stimulation IEEE Trans. Biomed. Eng. 58 310-20

De Geeter N, Crevecoeur G, Dupré L, Van Hecke W and Leemans A 2012 A DTI-based model for TMS using the independent impedance method with frequency-dependent tissue parameters Phys. Med. Biol. 57 2169-88

De Geeter N, Dupré L and Crevecoeur G 2016 Modeling transcranial magnetic stimulation from the induced electric fields to the membrane potentials along tractography-based neural fibers J. Neural Eng. 13026028

Forster M, Hattingen E, Senft C, Gasser T, Seifert V and Szelényi A 2011 Navigated transcranial magnetic stimulation and functional magnetic resonance imaging: advanced adjuncts in preoperative planning for central region tumors Neurosurgery 68 1317-24

Friston K, Ashburner J, Kiebel S, Nichols T and Penny W 2007 Statistical Parametric Mapping: The Analysis of Functional Brain Images (San Diego, CA: Academic)

Gabriel S, Lau R and Gabriel C 1996 The dielectric properties of biological tissues: III. Parametric models for the dielectric spectrum of tissues Phys. Med. Biol. 41 2271-93

Hernandez-Pavon J, Mäkelä N, Lehtinen H, Lioumis P and Mäkelä J 2014 Effects of navigated TMS on object and action naming Front. Hum. Neurosci. 8660 
Krieg S, Sabih J, Bulubasova L, Obermueller T, Negwer C, Janssen I, Shiban E, Meyer B and Ringel F 2014 Preoperative motor mapping by navigated transcranial magnetic brain stimulation improves outcome for motor eloquent lesions Neuro-Oncology 16 1274-82

Leemans A, Jeurissen B, Sijbers J and Jones D 2009 ExploreDTI: a graphical toolbox for processing, analyzing, and visualizing diffusion MR data Proc. Int. Society for Magnetic Resonance in Medicine (Hawaii, USA)

Lioumis P, Kičić D, Savolainen P, Mäkelä J and Kähkönen S 2009 Reproducibility of TMS-evoked EEG responses Hum. Brain Mapp. 30 1387-96

Lioumis P, Zhdanov A, Mäkelä N, Lehtinen H, Wilenius J, Neuvonen T, Hannula H, Deletis V, Picht T and Mäkelä J 2012 A novel approach for documenting naming errors induced by navigated transcranial magnetic stimulation J. Neurosci. Methods 204 349-54

Manola L, Holsheimer J, Veltink P and Buitenweg J 2007 Anodal versus cathodal stimulation of motor cortex: a modeling study Clin. Neurophysiol. 118 464-74

Nagarajan S, Durand M and Warman E 1993 Effects of induced electric fields on finite neuronal structures: a simulation study IEEE Trans. Biomed. Eng. 40 1175-88

Ojemann G, Creutzfeldt O, Lettich E and Haglund M 1988 Neuronal activity in human lateral temporal cortex related to short-term verbal memory, naming and reading Brain 111 1383-403

Orrison W 2008 Atlas of Brain Function (New York: Thieme)

Penfield W and Rasmussen T 1950 The Cerebral Cortex of Man (New York: Macmillan)

Picht $\mathrm{T}$ et al 2013 A comparison of language mapping by preoperative navigated transcranial magnetic stimulation and direct cortical stimulation during awake surgery Neurosurgery 72 808-19

Picht T, Schmidt S, Brandt S, Frey D, Hannula H, Neuvonen T, Karhu J, Vajkoczy P and Suess O 2011 Preoperative functional mapping for rolandic brain tumor surgery: comparison of navigated transcranial magnetic stimulation to direct cortical stimulation Neurosurgery 6 9581-8

Pouratian N and Bookheimer S 2010 The reliability of neuroanatomy as a predictor of eloquence: a review Neurosurg. Focus 28 E3

Roth B and Basser P 1990 A model of the stimulation of a nerve fiber by electromagnetic induction IEEE Trans. Biomed. Eng. 37 588-97

Salvador R 2009 Numerical modelling in transcranial magnetic stimulation PhD Thesis University of Lisbon, Portugal

Tournier J, Mori S and Leemans A 2011 Diffusion tensor imaging and beyond Magn. Reson. Med. $651532-56$

Tzourio-Mazoyer N, Landeau B, Papathanassiou D, Crivello F, Etard O, Delcroix N, Mazoyer B and Joliot M 2002 Automated anatomical labeling (AAL) of activations in SPM using a macroscopic anatomical parcellation of the MNI MRI single-subject brain NeuroImage 15 273-89

Wesselink W, Holsheimer J and Boom H 1999 A model of the electrical behaviour of myelinated sensory nerve fibres based on human data Med. Biol. Eng. Comput. 37 228-35 